\title{
The systematics of neutron reaction cross sections
}

\author{
Jimin Wang ${ }^{1, *}, X i$ Tao $^{1}$, Xiaolong Huang ${ }^{1}$, and Zhigang $\mathrm{Ge}^{1}$ \\ ${ }^{1}$ China Nuclear Data Center, China Institute of Atomic Energy, Beijing 102413, China
}

\begin{abstract}
The parameterized theoretical formulae of excitation functions for $(n, 2 n)$ and $(n, \gamma)$ reactions have been established, and for $(n, t o t),(n, n o n),(n, 3 n),(n, p),(n, d),(n, t),\left(n,{ }^{3} \mathrm{He}\right)$ and $(n, \alpha)$ have been recommended. According to these formulae, the SEF code have been developed for systematics calculation of these reactions. The calculated results with the systematics of the corresponding reactions of the discretional nucleus can be provided by the SEF code in the applied range quickly. At the same time, the comparison of calculated results with experimental and evaluated data can be given graphically.
\end{abstract}

\section{Introduction}

The cross sections of neutron-induced reactions are important for nuclear science and technology. The evaluation of nuclear reaction cross sections are based on experimental measurement, theoretical calculation and systematics.

All over the world, the experimental measurement has never been abandoned, and the progress is significant. However, the available measured data are scarce and scattered for some nuclei, reactions or energy regions.

Based on the nuclear models, some codes $[1,2]$ have been developed and used to calculate the cross sections. However, there are obvious discrepancy in the unmeasured energy regions.

Generally, the systematics is convenient and reliable for prediction of the neutron-induced reaction cross sections, compared to model theory calculations when experimental data are scarce.

In the present work, the parameterized theoretical formulae for $(n, t o t),(n, n o n),(n, 2 n),(n, 3 n),(n, \gamma),(n, p)$, $(n, d),(n, t),\left(n,{ }^{3} \mathrm{He}\right)$ and $(n, \alpha)$ have been established or recommended, and the SEF code has been developed for systematics calculation.

\section{Formulae}

Based on the constant temperature evaporation model and exciton model, taking the competition of other reactions and the contribution of pre-equilibrium emission into account, under some assumptions and approximations, the parameterized theoretical formulae of excitation functions for $(n, 2 n)$ and $(n, \gamma)$ reactions have been established. Only the most sensitive parameters are included in the formulae. For getting the parameters, the available experimental data of these reactions were analyzed and fitted by means of the nonlinear least square method. The fitted results agree fairly well with the measured data at some energy

*e-mail: jmwang@ciae.ac.cn and nuclei region. On the basis of the parameters of every nucleus, the correlations between the parameters and some quantity of the target nucleus can be expressed as simple functions. Using the regional parameters, more accurate systematics prediction for unmeasured nucleus or energy range can be provided. The parameterized theoretical formulae of $(n, t o t),(n, n o n),(n, 3 n),(n, p),(n, d)$, $(n, t),\left(n,{ }^{3} \mathrm{He}\right)$ and $(n, \alpha)$ have been recommended. In the formulae below, $A$ is the mass numbers of target nucleus, $E_{n}$ is the incident energy of neutron.

\subsection{Neutron total cross sections}

For $(n, t o t)$ reaction cross sections, the systematics of R.W.Bauer et al. [3, 4] and S.M.Grimes et al. [5, 6] are used. Using the assumptions of the Ramsauer model, the neutron total cross section can be expressed as:

$$
\sigma_{\text {tot }}=2 \pi(R+\lambda)^{2}(1-\alpha \cos \beta)
$$

where, the unit of cross section is b, $R$ is the radius of the nucleus, $t$ is the reduced wave length of neutron, $\alpha$ is a parameter with the magnitude between 0 and 1 , and $\beta$ denotes the phase difference between the wave that passes through the nucleus and the wave that goes around the nucleus.

$$
\begin{array}{r}
\beta=c A^{1 / 3} \cdot\left\{\left[\sqrt{a+b E_{n}}-\sqrt{E_{n}}\right]\right. \\
\left.+k^{\prime}\left[\sqrt{a+b E_{n}}-\sqrt{E_{n}}\right]^{2}\right\}
\end{array}
$$

The parameters $a, b, c$ and $k^{\prime}$ listed in Table 1 are taken from Ref. [6], the limited mass range is $7 \leqslant A$ and the limited energy range is from 6 to $60 \mathrm{MeV}$. These parameters have been obtained by fitting total neutron cross sections with the nuclear Ramsauer model for mass number $A>40$ and for neutron energies between 6 and $60 \mathrm{MeV}$, are extended to nuclei of mass $A<40$ [6]. 
Table 1. The parameters $a, b, c, k^{\prime}[6]$

\begin{tabular}{cccc}
\hline Parameter & $7 \leqslant A \leqslant 20$ & $20<A<40$ & $A>40$ \\
\hline$a$ & $41.0 \pm 0.5$ & $42.0 \pm 0.5$ & $38.0 \pm 0.5$ \\
$b$ & $0.67 \pm 0.01$ & $0.67 \pm 0.01$ & $0.85 \pm 0.01$ \\
$c$ & $0.425 \pm 0.01$ & $0.425 \pm 0.01$ & $0.46 \pm 0.01$ \\
$k^{\prime}$ & $0.07 \pm 0.002$ & $0.07 \pm 0.002$ & $0.07 \pm 0.002$ \\
\hline
\end{tabular}

\subsection{Non-elastic cross sections}

For $(n, n o n)$ reaction cross sections, the systematics of A.Chatterjee et al. [7] for neutron energy range from 1 to $50 \mathrm{MeV}$ are used.

$$
\begin{array}{r}
\sigma_{\text {non }}=\left(31.05 A^{-1 / 3}-25.91\right) E_{n}+342 A^{1 / 3} \\
+21.89 A^{2 / 3}+\left(0.223 A^{4 / 3}+0.673 A^{2 / 3}+617.4\right) / E_{n}
\end{array}
$$

where, the unit of cross section is mb and the unit of incident neutron energy is $\mathrm{MeV}$.

\section{$2.3(n, 2 n)$ and $(n, 3 n)$ reaction cross sections}

For $(n, 2 n)$ reaction cross sections, based on the constant temperature evaporation model taking the competition of $(n, 3 n)$ reaction and the contribution of preequilibrium emission into account, the systematics formulae of $(n, 2 n)$ reaction excitation function [8] have been established from threshold energy to $30 \mathrm{MeV}$ in the mass region $45 \leqslant A \leqslant 210$.

$$
\begin{array}{r}
\sigma_{n, 2 n}=\sigma_{n e} \frac{\sigma_{n, M}}{\sigma_{n e}}\left\{(1-\delta)\left[\left(\frac{\sigma_{n, 2 n}}{\sigma_{n, M}}\right)_{e q}-\left(\frac{\sigma_{n, 3 n}}{\sigma_{n, M}}\right)_{e q}\right]\right. \\
\left.+\delta\left[\left(\frac{\sigma_{n, 2 n}}{\sigma_{n, M}}\right)_{\mathrm{pre}}-\left(\frac{\sigma_{n, 3 n}}{\sigma_{n, M}}\right)_{\mathrm{pre}}\right]\right\}
\end{array}
$$

where, the unit of cross section is $\mathrm{b}, \sigma_{n e}$ is the nonelastic cross section from empirical formula [9], $\sigma_{n, M}$ is the neutron emission cross section of compound nucleus,

$$
\sigma_{n, M}=\sigma_{n, n^{\prime}}+\sigma_{n, 2 n}+\sigma_{n, 3 n}+\cdots \cdots
$$

$\delta$ denotes the contribution of preequilibrium emission, the subscript eq is the equilibrium cross section can be calculated by the evaporation model and subscript pre is the preequilibrium cross section can be calculated by the exciton model.

For the expressions of $(n, 3 n)$ reaction cross sections are similar to equation (4). In the expressions of $(n, 2 n)$ and $(n, 3 n)$ reaction cross sections, there are two ajustable parameters, the nuclear temperature $T$ and the ratio $\frac{\sigma_{n, M}}{\sigma_{n e}}$.

\subsection{Neutron capture cross sections}

The $(n, \gamma)$ reaction cross sections $[10,11]$ can be written as

$$
\sigma_{n, \gamma}=\sigma_{n, \gamma}(s)+\sigma_{n, \gamma}(d)
$$

$\sigma_{n \gamma}(s)$ is the contribution of statistical process and $\sigma_{n \gamma}(d)$ is the contribution of interaction between direct and semidirect process.
Based on the evaporation model and under some assumptions and approximations, $\sigma_{n \gamma}(s)$ can be expressed as

$$
\begin{array}{r}
\sigma_{n, \gamma}(s)=\left\{640 \alpha \sum_{L=0}^{L_{m}}(2 L+1) \frac{\exp \left(-1.346 b_{L}^{0.42}\right)}{E_{n}}\right\} \\
\times \frac{\exp \frac{S_{n}}{T}}{\exp \frac{S_{n}}{T}+\left[\exp \frac{S_{n}}{T}-1\right]^{2}}
\end{array}
$$

where, the unit of cross section is mb, the unit of incident neutron energy is $\mathrm{KeV}, b_{L}$ can be expressed as

$$
b_{L}=\frac{0.0158 \alpha}{\beta V_{L} \sqrt{E_{n}}}
$$

$V_{L}$ is the penetration factor of $L$ partial wave. There are two ajustable parameters $\alpha$ and $\beta$.

If we only consider the preequilibrium emission in the first step in the equilibrium process, which is characterized by excitions $n=3, \sigma_{n \gamma}(d)$ can be expressed as

$$
\sigma_{n, \gamma}(d)=C_{\gamma} \frac{\int_{0}^{E_{n}+S_{n}} \frac{E_{\gamma}^{4}}{\left(E_{\gamma}^{2}-E_{R}^{2}\right)^{2}+E_{\gamma}^{2} \Gamma_{R}^{2}} d E_{\gamma}}{E_{n}^{3}\left[1+0.035 A\left(1+\frac{S_{n}}{E_{n}}\right)^{3}\right]}
$$

where, the unit of incident neutron energy is $\mathrm{MeV}, E_{R}$ and $\Gamma_{R}$ are the giant dipole resonance parameters. There is only one ajustable parameter $C_{\gamma}$.

In Eqs. (7) and (9), $S_{n}$ is neutron separation energy for compound system. The parameter $\alpha$ can be determined by the systematics of [12] around $25 \mathrm{KeV}$. The fitting to Eq. (9) has been carried out with the collected $(n, \gamma)$ reaction cross sections for about forty nuclei, the systematic feature of parameter $C_{\gamma}$ has been obtained, can be expressed as

$$
C_{\gamma}=\exp \left(3.77+0.0389 A+0.000111 A^{2}\right)
$$

The fitting to Eqs. (7) and (8) have been carried out with the collected $(n, \gamma)$ reaction cross sections. For odd-A nuclei, the systematic feature of parameter $\beta$ have been obtained from about forty nuclei, can be expressed as

$$
\begin{array}{r}
\beta=\exp \left(-134+6.33 A-0.108 A^{2}+8.77 A^{3}\right. \\
\left.-3.44 \times 10^{-6} A^{4}+4.81 \times 10^{-9} A^{5}\right)
\end{array}
$$

For even-even nuclei, the systematic feature of parameter $\beta$ have been obtained from about fifty nuclei, can be expressed as

$$
\begin{array}{r}
\beta=\exp \left(-24.6-1.01 A-0.0207 A^{2}\right. \\
+19.8 \times 10^{-5} A^{3}-8.70 \times 10^{-7} A^{4} \\
\left.-1.35 \times 10^{-9} A^{5}\right)
\end{array}
$$

\section{5 (n,charged particle) reaction cross sections}

For $(n, x)\left[x=p, \alpha, d, t,{ }^{3} \mathrm{He}\right]$ reaction cross sections, the systematics of Zhao Zhixiang et al. [13,14] are used. The equilibrium cross sections are calculated by the evaporation model and the preequilibrium cross sections by the 
exciton model. The preequilibrium emission in the first step in the equilibrium process are considered, which is characterized by excitions $n=3$.

For $(n, x)[x=p, \alpha]$, it can be written as

$$
\sigma_{n, x}=C_{x}\left(\Delta_{1}^{x}+\frac{\lambda_{2}}{L_{n}} \frac{\Delta_{2}^{x}}{1+\Gamma_{n} / \Gamma_{x}}\right) /\left(1+\frac{\lambda_{2}}{L_{n}}+\frac{L_{x}}{L_{n}}\right)
$$

For $(n, x)\left[x=d, t,{ }^{3} \mathrm{He}\right]$, it can be written as

$$
\sigma_{n, x}=C_{x}\left(\frac{L_{x} / L_{n}}{\lambda_{2} / L_{n}}+\Gamma_{x} / \Gamma_{n}\right)
$$

In Eqs.(13) and (14), there are two adjustable parameters, $E_{c}^{x}$ and $C_{x} . E_{c}^{x}$ represents the generalized height of coulomb barrier and $C_{x}$ is a constant proportional to the maximum of the cross sections. For $(n, p)$ and $(n, \alpha)$ reactions in mass region $23 \leqslant A \leqslant 197$,

$$
\begin{gathered}
E_{c}^{p}=\left\{0.25 Z-0.6-0.001 Z^{2}\right. \\
\left.-2 \exp \left[-0.05(Z-28)^{2}\right]\right\} \\
\quad \times \exp \left[29.6(N-Z) / A^{3 / 2}\right] \\
C_{p}=\left(1+A^{1 / 3}\right)^{2} \exp \left(5.88-33.7 \frac{N-Z}{A}\right. \\
\left.-16.8 A^{-2 / 3}\right) \\
E_{c}^{\alpha}=-3.4+0.57 Z-0.003 Z^{2} \\
-3 \exp \left[-0.3(Z-28)^{2}\right] \\
C_{\alpha}=\left(1+A^{1 / 3}\right)^{2} \exp \left(2.0-23.7 \frac{N-Z}{A}\right. \\
\left.+21.0 A^{-2 / 3}\right)
\end{gathered}
$$

For $(n, d),(n, t)$ and $\left(n,{ }^{3} H e\right)$ reactions, $E_{c}^{d}$ and $E_{c}^{t}$ are replaced by $E_{c}^{p}, E_{c}^{3} H e$ is replaced by $E_{c}^{\alpha}$.

$$
\begin{gathered}
C_{d}=23\left(1-0.052 Z+0.00083 Z^{2}\right) \\
C_{t}=5.81\left(1-0.052 Z+0.00083 Z^{2}\right) \\
C_{{ }^{3} \mathrm{He}}=2.9\left(1-0.052 Z+0.00083 Z^{2}\right)
\end{gathered}
$$

\section{SEF code}

Based on the established and recommended systematics formulae and the regional parameters, the SEF code was developed to calculate the cross sections of the neutroninduced reaction. Figure 1 shows the flow chart of SEF code, $A$ is the mass numbers and $Z$ is the number of protons for target nuclide, $P$ represents the reaction channels, $E_{n}$ is the incident energy of neutron and $C S$ is the value of the cross sections. Figure 2 and 3 shows the results of calculation for ${ }^{208} \mathrm{~Pb}(n, 2 n)$ and ${ }^{208} \mathrm{~Pb}(n, \gamma)$ with SEF code.

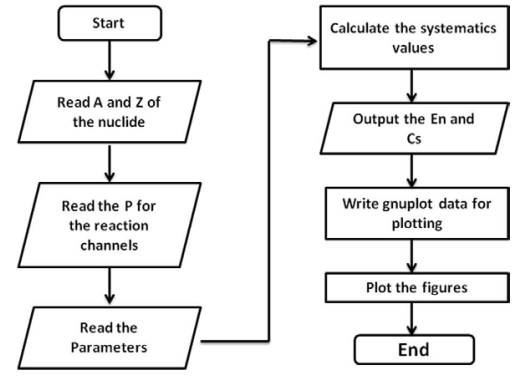

Figure 1. The flow chart of SEF code

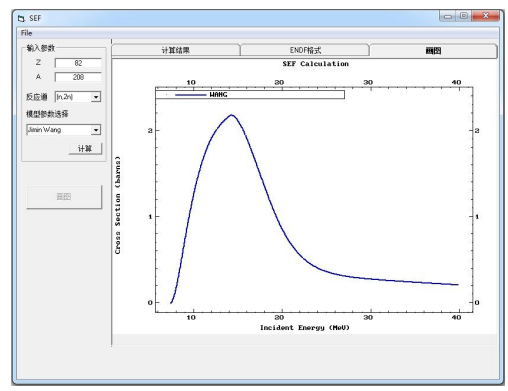

Figure 2. The calculated results of ${ }^{208} P b(n, 2 n)$ with SEF code

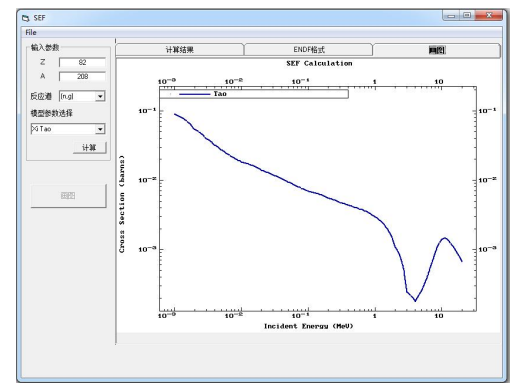

Figure 3. The calculated results of ${ }^{208} P b(n, \gamma)$ with SEF code

\section{Results and discussion}

The results of systematics for the corresponding reactions of the discretional nucleus can be provided by the SEF code in the limitted mass number and neutron energy range. From Figures 4 to 10, the comparisons of experimental data taked from EXFOR [15], evaluated value and results of systematics for $(n$, tot $),(n, n o n),(n, 2 n),(n, 3 n)$, $(n, \gamma),(n, p),(n, \alpha),(n, d),(n, t)$ and $\left(n,{ }^{3} \mathrm{He}\right)$ reaction cross sections are shown respectively. The results indicated that the predicted cross sections are consistent with the measured and evaluated data within the errors for $(n, t o t)$, $(n, n o n),(n, 2 n),(n, 3 n),(n, \gamma),(n, p)$ and $(n, \alpha)$ reactions. For $(n$, tot $),(n, n o n),(n, 2 n)$ and $(n, 3 n)$ reactions, the energy range is extended to more than $20 \mathrm{MeV}$. Hence more accurate systematics prediction for unmeasured nuclei or neutron energy ranges can be provided. The agreement between the predicted curves and experimental data is fair for $(n, d),(n, t)$ and $\left(n,{ }^{3} \mathrm{He}\right)$ reactions. So, further research is needed for the systematics of these reactions. 


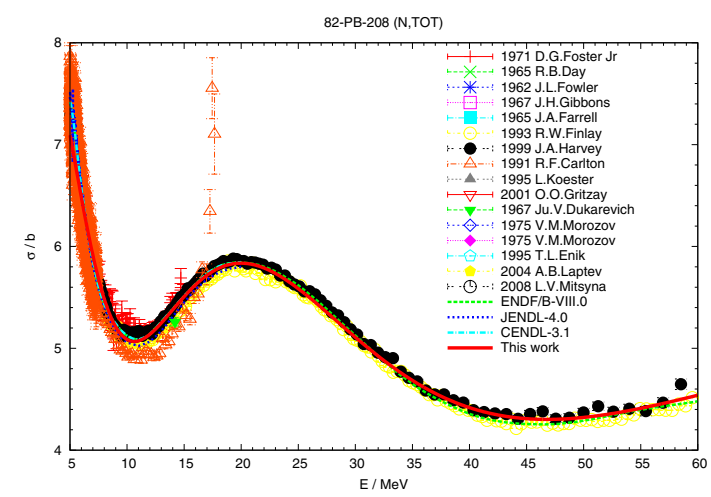

Figure 4. The comparison of the results of systematics with experimental and evaluated data for ${ }^{208} \mathrm{~Pb}(n$, tot $)$

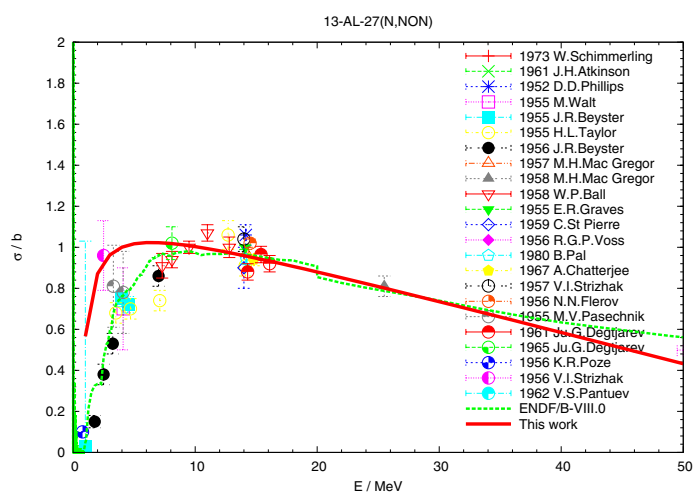

Figure 5. The comparison of the results of systematics with experimental and evaluated data for ${ }^{27} \mathrm{Al}(n$, non $)$

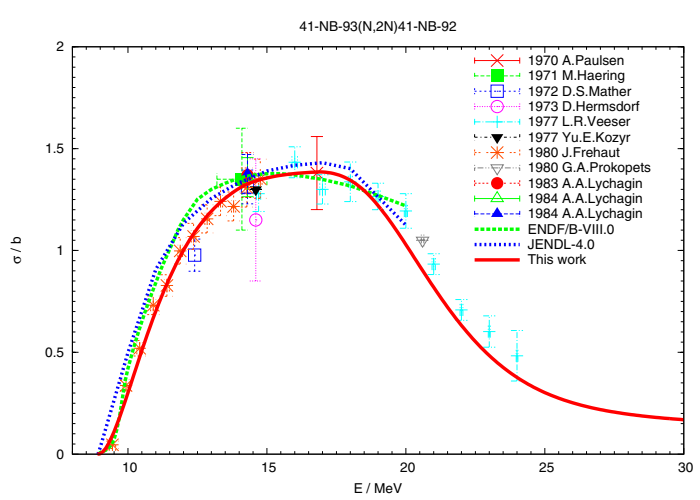

Figure 6. The comparison of the results of systematics with experimental and evaluated data for ${ }^{93} \mathrm{Nb}(n, 2 n)$

\section{Acknowledgements}

This work was supported by national nature science foundation of China (No. 11790320), the Continuous Basic Scientific Research Project (No. WDJC-2019-09).

\section{References}

[1] M.Herman, R.Capote, B.V.Carlson, P.Oblozinsky, M.Sin, A.Trkov, H.Wienke, V.Zerkin, Nucl. Data Sheets 108, 2655 (2007)

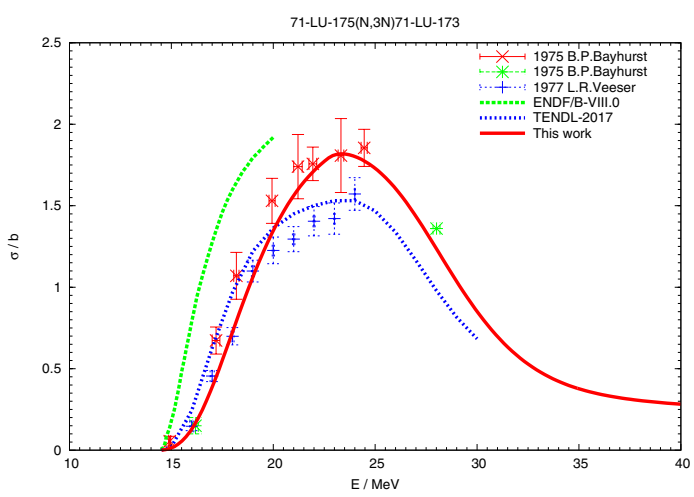

Figure 7. The comparison of the results of systematics with experimental and evaluated data for ${ }^{175} L u(n, 3 n)$

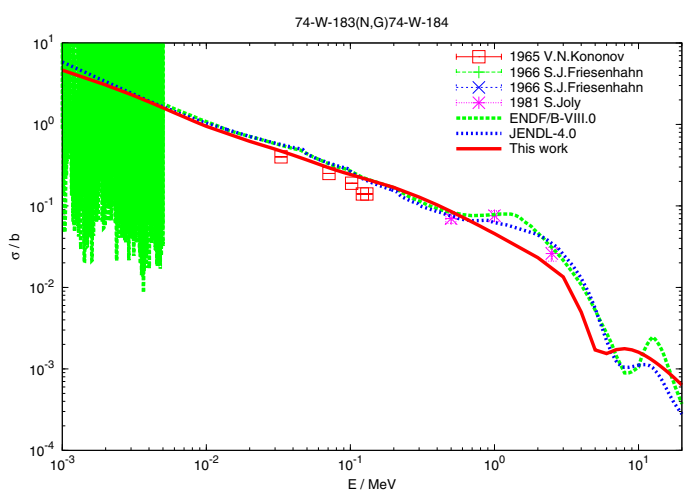

Figure 8. The comparison of the results of systematics with experimental and evaluated data for ${ }^{183} W(n, \gamma)$

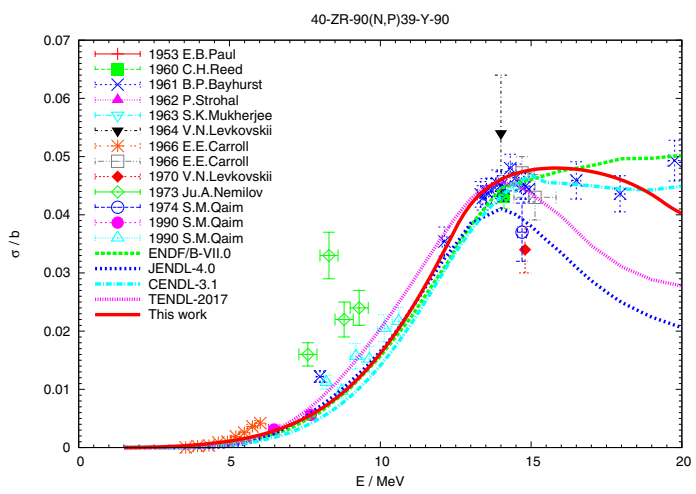

Figure 9. The comparison of the results of systematics with experimental and evaluated data for ${ }^{90} \mathrm{Zr}(n, p)$

[2] A.J.Koning and D.Rochman, Nucl. Data Sheets 113, 2841 (2012)

[3] R.W.Bauer, J.D.Anderson, S.M.Grimes, V.A.Madsen, Italian Physical Society 59, 317 (1997)

[4] R.W.Bauer, J.D.Anderson, S.M.Grimes, D.A.Knapp, V.A.Madsen, Nucl. Sci. Eng. 130, 348 (1998)

[5] S.M.Grimes, J.D.Anderson, R.W.Bauer, V.A.Madsen, Nucl. Sci. Eng. 130, 340 (1998)

[6] S.M.Grimes, J.D.Anderson, R.W.Bauer, Nucl. Sci. Eng. 135, 296 (2000) 


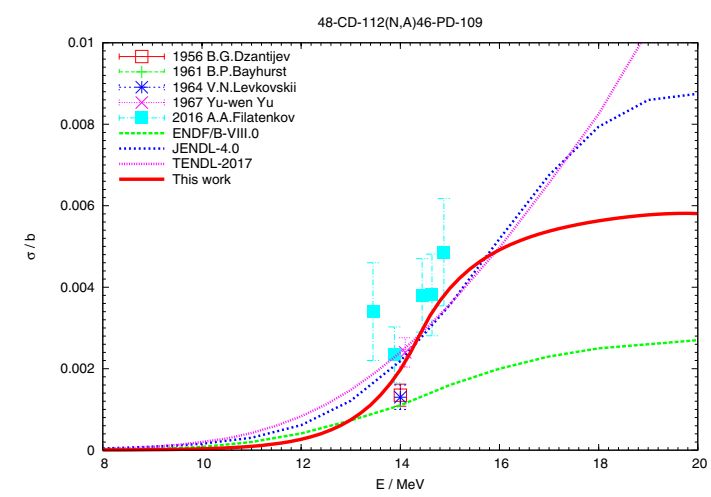

Figure 10. The comparison of the results of systematics with experimental and evaluated data for ${ }^{112} C d(n, \alpha)$

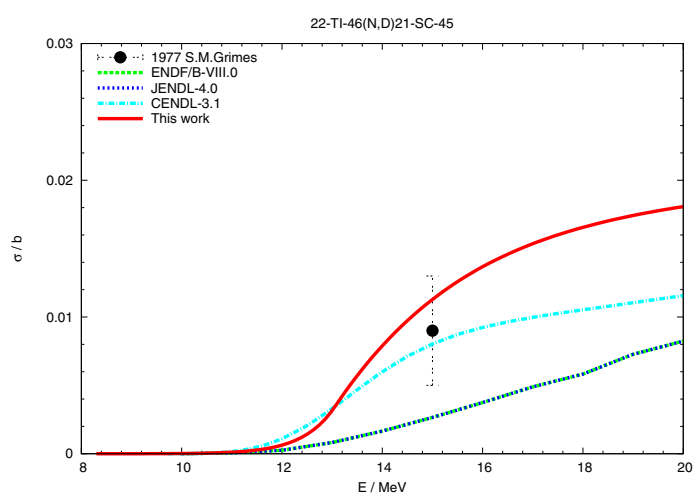

Figure 11. The comparison of the results of systematics with experimental and evaluated data for ${ }^{46} \mathrm{Ti}(n, d)$

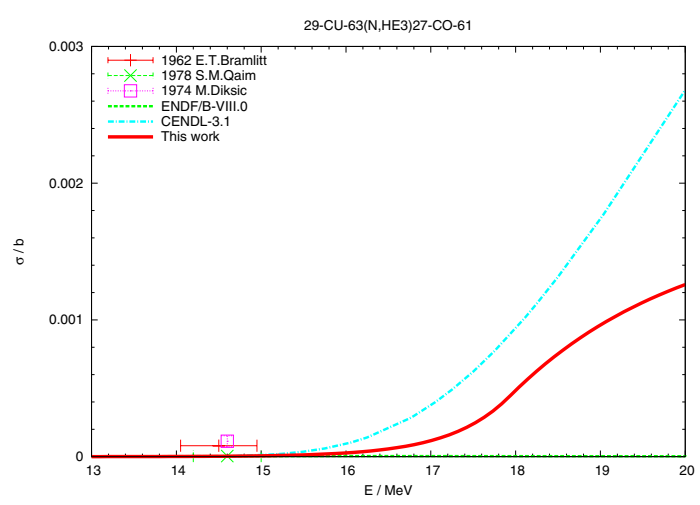

Figure 13. The comparison of the results of systematics with experimental and evaluated data for ${ }^{63} \mathrm{Cu}\left(n,{ }^{3} \mathrm{He}\right)$

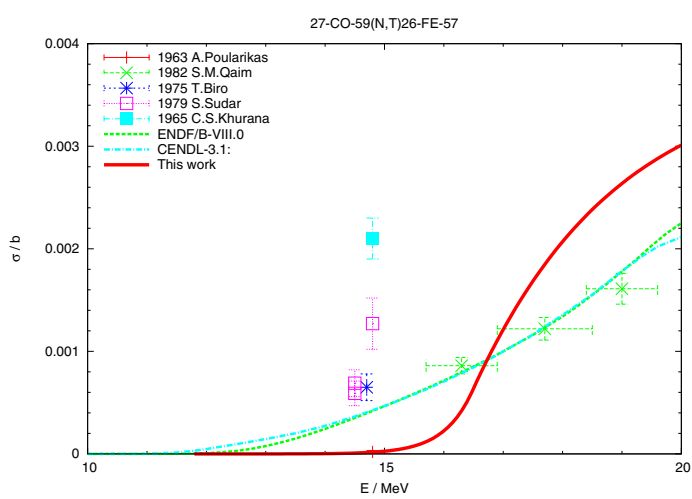

Figure 12. The comparison of the results of systematics with experimental and evaluated data for ${ }^{59} \mathrm{Co}(n, t)$

[7] A.Chatterjee, K.H.N.Murthy and S.K.Gupta, INDC(IND)-27/GJ (1980)

[8] Ji-Min Wang, Xi Tao, Xiao-Long Huang, You-Xiang Zhuang, Nucl. Instrum. Methods in Physics Res., Sect. B 268, 2221 (2010)

[9] W.G. Davey et al., ANL 75, 34 (1975)

[10] Zhaozhixiang, Zhoudelin, Caidunjiu, Chin. Jour. of Nucl. Phys. 11, 71 (1989)

[11] Zhaozhixiang, Zhoudelin, Chin. Jour. of Nucl. Phys. 13, 139 (1991)

[12] Zhaozhixiang et al., Proc. Conf. on Nucl. Data for Sci. and Tech. 513, Mito, Japan (1988)

[13] Zhaozhixiang and Zhoudelin, Nucl. Sci. and Eng. 99, 367 (1988)

[14] Zhaozhixiang and Zhoudelin, Chin. Jour. of Nucl. Phys. 10, 337 (1988)

[15] N.Otuka et al., Nucl. Data Sheets 120, 272 (2014) 